\title{
Rendimiento académico de estudiantes de nuevo ingreso en Licenciaturas e Ingenierías de la Facultad Multidisciplinaria de Ilobasco, Universidad Católica de El Salvador, durante el ciclo II-2013
}

\author{
Héctor Raúl Zeledón ${ }^{1}$, \\ Willian Edgardo Ayala ${ }^{2}$
}

Recepción: 10-10-2014 / Aceptación: 04-02-2014

\section{Resumen}

Se estudió el rendimiento académico de los alumnos de nuevo ingreso, inscritos en ciclo II 2013 de Licenciaturas e Ingenierías de la Facultad Multidisciplinaria de Ilobasco de la Universidad Católica de El Salvador. Se establecieron índices de aprobación y reprobación, a través de una comparación por carrera, sexo e institución educativa donde cursaron bachillerato. Además se presentan el listado de asignaturas impartidas en el semestre, con sus respectivos porcentajes de aprobación y reprobación.

Para la obtención de la información se utilizaron las calificaciones proporcionadas por registro académico de la Universidad, en la cual se identificó a los alumnos que reprobaron asignaturas, aplicándoseles una entrevista escrita, a fin de detectar las principales causas de este fenómeno.

En términos generales, se encontró que el rendimiento académico aumenta en el transcurso del ciclo en un $27.4 \%$ para el sexo masculino; mientras que para el sexo femenino lo hace en un 3.9\%. Por otra parte el $31.3 \%$ de los alumnos reprobaron al menos una asignatura.

Palabras Clave: Rendimiento académico, proceso de enseñanza aprendizaje, asignatura, calificaciones, reprobación, aprobación

\begin{abstract}
It was studied the performance of new freshmen students enrolled in the term II-2013 of bachelor and engineering degree of the Multidisciplinary Faculty of Ilobasco of Universidad Catolica de El Salvador. Ranges of passing and failing were established through a comparison by career, sex and educational institution where they studied high-school. Besides, it presented the list of subjects taught during the semester with their respective range of passing and failing.

In order to get the information, the grades provided by the academic registrar of the university were used. It identified the students that flunked subjects by administering a written interview with the purpose to find out the main causes of this problem.

In general terms, it was found that the academic performance increases along the semester in $27.4 \%$ for the male students; while the female students increases it in 3.9\%. On the other hand, $31.3 \%$ of the students flunked one subject at least.
\end{abstract}

Key words: Academic performance, teaching-learning process, subject, grades, passing, failing

1. Máster en Asesoría Educativa, docente-investigador, Facultad Multidisciplinaria de Ilobasco, hector.zeledon@ catolica.edu.sv

2. Máster en Asesoría Educativa, docente-investigador, Facultad Multidisciplinaria de Ilobasco, willian.ayala@ catolica.edu.sv

ISSN 2311-5513, CE, febrero 2015 $\quad$ Volumen 2 


\section{Introducción}

La Ley de Educación Superior de El Salvador, en el Art. 23, establece que todas las universidades deben contar con una unidad de Registro Académico, en la que se archiven los documentos generales del alumnado, así como aspectos generales sobre su rendimiento dentro de la institución. El registro académico se define como "el producto de asimilación del contenido de los programas de estudio, expresado en calificaciones dentro de una escala convencional" (Figueroa, 2004). Esta escala de calificaciones es definida por cada institución, y en última instancia, es la que determina la aprobación o reprobación de un alumno dentro de un curso o asignatura.

El rendimiento académico de los alumnos no solo se ve influenciado por un factor, si no por muchas variables, las cuales determinan su aprendizaje. Todo proceso educativo debe buscar constantemente el perfeccionamiento del alumno y, por consecuencia, la mejora del rendimiento académico; de ahí la relevancia y necesidad de que la Universidad considere estas en el aprendizaje de los alumnos.

Conocer todas las posibles variables ofrece oportunidades de mejora y evita que muchos alumnos terminen reprobando asignaturas y/o retirándose de la universidad. Sin lugar a dudas

Al final de cada ciclo el rendimiento académico de los alumnos no es el producto de una única capacidad, sino el resultado sin- tético de una serie de factores que actúan en, y desde, la persona que aprende. Las variables y los indicadores que inciden en él se clasifican de diversas maneras, a saber: variables demográficas o de identificación (sexo, edad, estado civil, experiencia laboral), variables académicas (tipos de estudios cursados, curso, opción en que se estudia una carrera, rendimiento previo), variables sociofamiliares (estudios de los padres, situación laboral de los mismos, lugar de residencia familiar, lugar de estudio) (Goméz, Oviedo, y Martinéz, 2011).

Dentro del rendimiento académico, el apoyo que la familia ofrece a los hijos en el proceso de enseñanza aprendizaje, influye en el rendimiento de los alumnos. Tanto así que, la experiencia realizada por Daugherty y Lane (1999) demostró que, los alumnos cuyos parientes habían estudiado en el mismo colegio, tenían más probabilidad de persistir en sus estudios y graduarse. Por otra parte, el modelo de deserción de Girón y González (2005) determinó que la probabilidad de abandonar los estudios se incrementaba cuando el estudiante no recibía apoyo de parte de su familia.

Ante un escenario así, surge un nuevo elemento: la voluntad del alumno por aprender. Este es un factor que juega un papel muy importante en el rendimiento académico, ya que si el alumno no posee el deseo y la motivación por adquirir conocimiento, aunque el docente le brinde todas las herramientas para hacerlo, él no aprenderá. 
La voluntad y la disposición por aprender, más que ser deseos o aficiones momentáneas, implican una serie de acciones por parte del alumno; acciones que lo lleven a cumplir con optimismo sus deberes académicos. Dentro de estas se pueden mencionar la asistencia, la participación en clases, la responsabilidad en la entrega de las actividades y; sobre todo, aplicar técnicas y hábitos de estudio.

Otro de los factores que tienen relación con el rendimiento académico de los estudiantes en la Universidad es la vocación. Sentir pasión por la carrera que estudian es fundamental para brindar obtener buenos resultados. Sin embargo, en ocasiones los alumnos eligen una determinada carrera sin tener ninguna orientación, ni seguridad de lo que quieren estudiar. Esto les lleva a brindar un rendimiento pobre, deficiente, reprobar materias y abandonar la misma carrera.

Pero si el alumno estudia lo que realmente le gusta, obtendrá mejores resultados en todos los aspectos: académico, personal, profesional, etc. Esta valoración se fundamente en el trabajo de Rodríguez, Fita y Torrado (2004), quienes encontraron que los alumnos que estudiaban una carrera de su elección o preferencia, obtenían una mayor tasa de rendimiento en comparación a quienes cursaban una carrera que no había sido su primera elección.

Es importante mencionar que el rendimiento académico previo, también juega un papel muy importante dentro de la vida académica del estudiante universitario. De acuerdo a Garvanzo (2007), “El rendimiento académico previo a la universidad constituye uno de los indicadores con mayor capacidad predictiva en el rendimiento académico en estudiantes universitarios y tiene mucho que ver la calidad educativa de la institución de la que proviene el estudiante".

Según Tejedor, (2003:10), este aspecto constituye una variable sintética, en la que concurren numerosos factores (aptitud del alumno, voluntad, esfuerzo, características de la enseñanza que ha recibido); y que no solo refleja el resultado del aprendizaje, sino que es una expresión - en cierto sentido - de toda la persona del alumno en cuanto estudiante. Esto es apoyado por Garvanzo (2007), cuando menciona que,

Los estudiantes también pueden ver afectado su rendimiento académico con aspectos relacionados con la universidad misma. Estudio realizado por (Salonava, Martínez, Bresó, Llorens, Gumbau S., Gumbau, Grau R. (2005) con estudiantes universitarios en la Universidad de Jaume I, indican que elementos como: condiciones de las aulas, servicios, plan de estudios y formación del profesorado, se presentan como obstaculizadores del rendimiento académico; que a su vez también pueden ser facilitadores. 


\section{Metodología}

Esta investigación se realizó durante el ciclo II del año 2013, con los estudiantes de nuevo ingreso de la Facultad Multidisciplinaria de Ilobasco de la Universidad Católica de el Salvador (UNICAES) ${ }^{3}$, en las carreras:

- Ingeniería en Sistemas Informáticos

- Licenciatura en Mercadeo y Negocios Internacionales

- Licenciatura en Ciencias de la Educación con especialidad del Idioma Inglés

- Licenciatura en Enfermería

Para estas carreras se estudiaron 16 diferentes asignaturas, entre materias comunes y asignaturas propias de los programas de estudio.
Los estudiantes de la Universidad Católica de El Salvador, Facultad Multidisciplinaria de Ilobasco provenían de diferentes institutos, ubicados en distintos departamentos de la Zona Central y Paracentral de El Salvador (ver figura 1).

Al analizar los resultados de la Prueba de Aprendizaje y Aptitudes para Egresados de Educación Media (PAES), realizada por el Ministerio de Educación de la República de El Salvador (MINED), se observaron notas bastante similares para los diferentes departamento: Para Cabañas, 4.6; Chalatenango, 4.8; Cuscatlán, 4.8 y San Vicente, 4.7. Estas cifras además de ser un parámetro para la educación el país en general, también fueron una referencia del rendimiento académico de los estudiantes, provenientes de esa zona del país previo a su ingreso a la Universidad.

\begin{tabular}{|c|c|}
\hline Zona Central & Zona Paracentral \\
\hline Chalatenango: municipio de Nombre de & Cabañas: municipios de: Ilobasco, San \\
Jesús; & Isidro, Sensuntepeque y Tejutepeque \\
\hline Cuscatlán: municipios de: Cojutepeque, & San Vicente: municipios de: \\
San Rafael Cedros y Suchitoto; & $\begin{array}{c}\text { Apastepeque, San Lorenzo, San Sebastián, } \\
\text { San Vicente, Santo Domingo y Verapaz }\end{array}$ \\
\hline
\end{tabular}

Figura 1. Lugares de residencia de los alumnos involucrados en el estudio.

3. Los autores también se referirán a esta institución bajo sus siglas. 
Al comparar los resultados de la prueba PAES del sector público a nivel nacional con las del sector privado, el primero obtuvo un promedio de 4.6; mientras que el promedio para el sector privado fue de 5.6. En la tabla 1, se puede observar que los estudiantes de la Universidad provenían en mayor porcentaje del sector público, cuyos resultados en la PAES fueron inferiores en comparación a los resultados del sector privado.

Tabla 1. Estudiantes por tipo de Centro Educativo y Departamento

\begin{tabular}{|c|c|c|}
\hline \multirow{2}{*}{ Departamento } & \multicolumn{2}{|l|}{ Tipo de Centro Educativo } \\
\cline { 2 - 3 } & Público & Privado \\
\hline Cabañas & 77 & 6 \\
\hline Chalatenango & 1 & 0 \\
\hline Cuscatlán & 7 & 9 \\
\hline San Vicente & 31 & 3 \\
\hline TOTAL & 116 & 18 \\
\cline { 2 - 3 }
\end{tabular}

Para realizar la investigación se tomaron en cuenta los índices de aprobación de los alumnos de nuevo ingreso de las carreras antes mencionadas, estableciendo las principales causas de reprobación de asignaturas y una comparación por carrera, sexo e instituto de procedencia; tipo de instituto y el record académico previo. El número de estudiantes por carrera y sexo, se muestra en la Tabla 2.
Tabla 2. Número de estudiantes de acuerdo

\begin{tabular}{|c|c|c|c|}
\hline \multicolumn{2}{|c|}{ a carrera y sexo } \\
\cline { 2 - 4 } Carrera & Masculino & Femenino & Total \\
\hline $\begin{array}{c}\text { Ingeniería en Sistemas } \\
\text { Informáticos. }\end{array}$ & 15 & 0 & 15 \\
\hline $\begin{array}{c}\text { Licenciatura en Mercadeo y } \\
\text { Negocios Internacionales. }\end{array}$ & 7 & 10 & 17 \\
\hline $\begin{array}{c}\text { Licenciatura en Ciencias de la } \\
\text { Educación con Especialidad En } \\
\text { Idioma Inglés. }\end{array}$ & 26 & 41 & 67 \\
\hline Licenciatura en Enfermería. & 3 & 32 & 35 \\
\hline TOTAL & $\mathbf{5 3}$ & $\mathbf{8 2}$ & $\mathbf{1 3 4}$ \\
\cline { 2 - 4 } & & & \\
\cline { 2 - 4 } & &
\end{tabular}

Los datos utilizados para la investigación fueron las calificaciones de los estudiantes de nuevo ingreso del año 2013; además de información personal de los estudiantes.

Cada ciclo dentro de la Universidad Católica de El Salvador (UNICAES) se desarrolla durante 20 semanas, divididas en tres periodos. Al finalizar cada periodo, los docentes de las diferentes asignaturas presentan a Registro Académico de la Universidad, un control de calificaciones el cual contiene las notas de todos los estudiantes, y el porcentaje de asistencia. Los promedios de cada periodo son calculados por el sistema informático de Registro Académico.

Para la recolección de las calificaciones, se solicitó, a Consejo Académico de la Universidad, acceso a los colectores correspondientes a los tres periodos del ciclo II - 2013, a fin de verificar las calificaciones de cada estudiante. Además, se elaboró un cuestionario que se utilizó a través de una entrevista para recabar datos de los estudiantes. 
Los colectores del período uno y dos contenían milésimas; mientras que el colector del periodo tres, décimas. Para calcular la calificación final de las asignaturas, según el Reglamento de Evaluación del Rendimiento Estudiantil de la UNICAES en el artículo 5, se aproximará a la décima inmediata superior cuando la centésima sea mayor o igual a cinco; mientras que para aprobar una asignatura (artículo 6), los estudiantes deben alcanzar un promedio final de 6.0, con al menos el $75 \%$ de asistencia en sus clases.

Al poseer los resultados correspondientes al primer periodo, se elaboraron estadísticas de porcentajes de aprobación y reprobación, utilizando los criterios de periodo, carrera, sexo y tipo de instituto de procedencia. Este proceso se realizó para los tres periodos y las calificaciones finales. Con base en las estadísticas, se comparó el rendimiento de las diferentes carreras en relación a la variable del sexo de los estudiantes.

Al finalizar el segundo periodo, se aplicó la técnica de la entrevista al $83.3 \%$ de los estudiantes que reprobaron una o más asignaturas, a fin de determinar porcentualmente las razones a las cuales ellos le atribuían su reprobación.

\section{Resultados}

Al consultar a los estudiantes sobre cuáles eran las causas de reprobación más frecuentes, el 40\% de ellos aseguró que este fenómeno se debía a la falta de conocimiento previo sobre las asignaturas; aunado a la dificultad para comprender las explicaciones del docente $(24 \%) y$ la falta de tiempo para dedicarlo explícitamente al estudio (20\%).

\subsection{Variables de análisis}

$\mathrm{Al}$ ver las cifras de los estudiantes reprobados dentro de las diferentes asignaturas, se clasificaron los resultados de acuerdo a las siguientes variables:

a. Demográficas: Sexo, edad, estado familiar, estado laboral.

b. Académicas: Rendimiento previo.

c. Socio Familiares: Nivel académico de padres, concepción familiar de la educación.

d. Actitudinales: Afinidad con la carrera, hábitos de estudio, autoconcepto.

e. Pedagógicas: Metodología del docente.

\section{a. Demográficas}

En estas variables se encontró que los porcentajes de reprobación para el sexo masculino fue más alto (47.1\%), que el del sexo femenino (21.7\%). En cuanto al indicador de edad, el 68\% de los sujetos estudiados oscilaron entre las edades de 17 a 19 años; mientras que el $32 \%$ se situó en edades mayores a los 20 años.

$\mathrm{Al}$ analizar el estado civil de los sujetos, la mayoría (98\%) afirmó ser soltero; y un $2 \%$ se encontraba en unión libre. Otro factor fue el 
estado laboral de los sujetos, de los cuales un 28\% manifestó estudiar y trabajar, mientras que el resto $(72 \%)$ solo estudia.

\section{b. Académicas}

Dentro de las variables académicas, se tomó el resultado de la PAES 2012 como referencia de rendimiento previo. El promedio obtenido por los alumnos dentro de esta prueba fue de 5.6 .

\section{c. Sociofamiliares}

Respecto a estas variables se tomó como parámetro el nivel académico de los padres (ver tabla 3).

\section{d. Actitudinales}

En cuanto a las variables actitudinales, el 24\% de los sujetos afirmó no se encontraba estudiando la carrera de su preferencia. Este dato llamó mucho la atención.

Por su parte, en el aspecto horario de estudio, el $68 \%$ de los entrevistados no tenía un espacio establecido para ellos. Esto podría justificar que el 32\% de los estudiantes consideren que no finalizarán de la mejor manera; mientras que un $2 \%$ aseguró tajantemente que no los culminará.

\section{e. Pedagógicas}

En este tipo de variables, el $24 \%$ afirmó no comprender las explicaciones del docente durante las clases. Por su parte, un 22\% de los alumnos dijeron que los docentes no siempre muestran apertura para responder consultas.

\subsection{Alumnos aprobados y reprobados}

Dentro del criterio de aprobado se tomó a los estudiantes que no habían reprobado ninguna de las asignaturas cursadas (Figura 2).

Tabla 3. Nivel Académico de padres de estudiantes reprobados

\begin{tabular}{|c|c|c|}
\hline Nivel Académico & Padre & Madre \\
\hline Educación Básica & $55.3 \%$ & $43.9 \%$ \\
\hline Educación Media & $21.1 \%$ & $26.8 \%$ \\
\hline Educación Técnica & $7.9 \%$ & $7.3 \%$ \\
\hline Educación Superior & $15.8 \%$ & $22.0 \%$ \\
\hline
\end{tabular}

Nota: Se expresan los resultados en porcentajes, de acuerdo al nivel académico de los padres de los alumnos reprobados en alguna asignatura. 


\section{Cantidad de alumnos aprobados y reprobados en el ciclo}

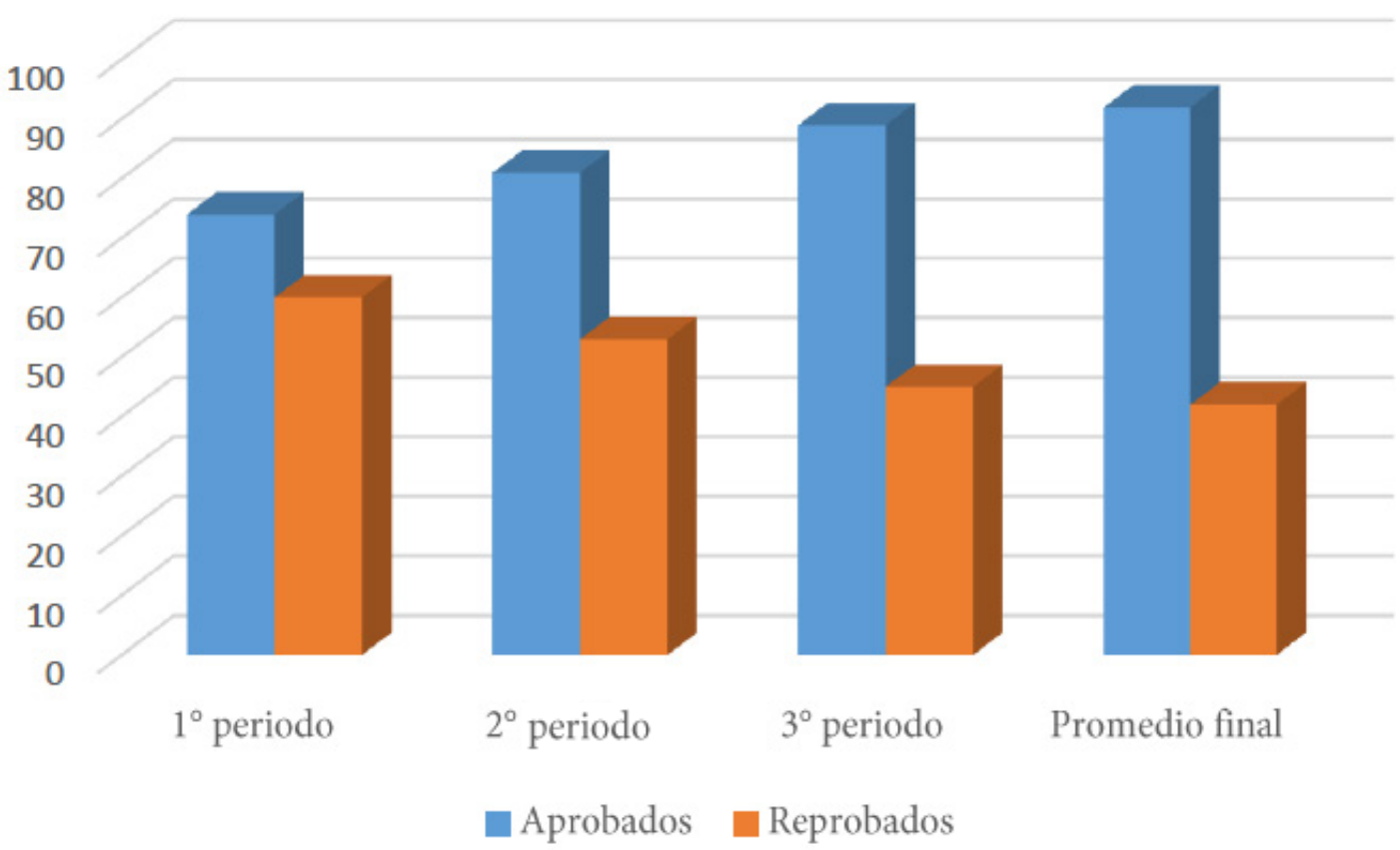

Al comparar los porcentajes del criterio periodo académico aprobado con la variable sexo se obtuvo:

Tabla 4. Porcentaje de estudiantes aprobados

\begin{tabular}{|c|c|c|c|c|}
\hline \multirow{2}{*}{ Periodo } & \multicolumn{2}{|c|}{ Aprobados } & \multicolumn{2}{c|}{ Reprobados } \\
\cline { 2 - 5 } & Masculino & Femenino & Masculino & Femenino \\
\hline Periodo Uno & $29.4 \%$ & $71.1 \%$ & $70.6 \%$ & $28.9 \%$ \\
\hline Periodo Dos & $43.1 \%$ & $71.1 \%$ & $56.9 \%$ & $28.9 \%$ \\
\hline Periodo Tres & $51.0 \%$ & $75.9 \%$ & $49.0 \%$ & $24.1 \%$ \\
\hline Promedio Final & $52.9 \%$ & $78.3 \%$ & $47.1 \%$ & $21.7 \%$ \\
\hline
\end{tabular}

Nota: Se realizó una comparación entre las variables de periodo de estudio y sexo de los estudiantes, tomando información de los colectores de notas, UNICAES (2013). 
Diferenciando las mismas variables, pero de forma individualiza por carrera, se obtuvo:

Tabla 5. Porcentaje de aprobación y reprobación de Ingeniería en Sistemas Informáticos

\begin{tabular}{|c|c|c|c|c|}
\hline \multirow{2}{*}{ Periodo } & \multicolumn{2}{|c|}{ Masculino } & \multicolumn{2}{c|}{ Femenino } \\
\cline { 2 - 5 } & Aprobados & Reprobados & Aprobados & Reprobados \\
\hline Uno & $0.0 \%$ & $100.0 \%$ & $0.0 \%$ & $0.0 \%$ \\
\hline Dos & $20.0 \%$ & $80.0 \%$ & $0.0 \%$ & $0.0 \%$ \\
\hline Tres & $33.3 \%$ & $66.7 \%$ & $0.0 \%$ & $0.0 \%$ \\
\hline Promedio Final & $20.0 \%$ & $80.0 \%$ & $0.0 \%$ & $0.0 \%$ \\
\hline
\end{tabular}

Nota: Se realizó una comparación entre las variables de periodo de estudio y sexo de los estudiantes, tomando información de los colectores de notas, UNICAES (2013).

En el caso de Ingeniería en Sistemas Informáticos, fue notable que no se inscribieran estudiantes del sexo femenino dentro de la carrera. Además se puedo observar que el porcentaje de aprobación aumentó en un $20 \%$ en el transcurso del ciclo.

Para la Licenciatura en Mercadeo y Negocios Internacionales se observó:

Tabla 6. Porcentaje de aprobación y reprobación de Licenciatura en Mercadeo y Negocios Internacionales

\begin{tabular}{|c|c|c|c|c|}
\hline \multirow{2}{*}{ Periodo } & \multicolumn{2}{|c|}{ Masculino } & \multicolumn{2}{c|}{ Femenino } \\
\cline { 2 - 5 } & Aprobados & Reprobados & Aprobados & Reprobados \\
\hline Uno & $14.3 \%$ & $85.7 \%$ & $40.0 \%$ & $60.0 \%$ \\
\hline Dos & $42.9 \%$ & $57.1 \%$ & $30.0 \%$ & $70.0 \%$ \\
\hline Tres & $42.9 \%$ & $57.1 \%$ & $30.0 \%$ & $70.0 \%$ \\
\hline Promedio Final & $57.1 \%$ & $42.9 \%$ & $40.0 \%$ & $60.0 \%$ \\
\hline
\end{tabular}

Nota: Se realizó una comparación entre las variables de periodo de estudio y sexo de los estudiantes, tomando información de los colectores de notas, UNICAES (2013).

En el sexo masculino, el porcentaje de aprobación aumentó un 42.9\% desde el primer periodo hasta la nota final. Asimismo, el sexo masculino presentó un porcentaje de aprobación final mayor que el sexo femenino, quienes obtuvieron un porcentaje de reprobación de $60 \%$. Al comparar estos resultados con los datos obtenidos para Ingeniería en Sistemas Informáticos, en el caso del sexo masculino, en ambas carreras hubo un aumento en el rendimiento académico a lo largo del ciclo. 
En el caso de Licenciatura en Ciencias de la Educación con Especialidad del Idioma Inglés (ver tabla 7).

En comparación con las carreras anteriores, en Licenciatura en Ciencias de la Educación con Especialidad del Idioma Ingles, el sexo femenino presentó porcentajes de aprobación más altos. A su vez, se evidenció una mejora porcentual promedio de $14 \%$ en el rendimiento académico.

Los resultados referentes a la Licenciatura en Enfermería se muestran en la Tabla 8.
En el caso de Licenciatura en Enfermería, el rendimiento fue uniforme. Aun así se notó una mejora del de $3.2 \%$ en el rendimiento académico de la población femenina.

Durante las dieciséis asignaturas cursadas por estudiantes de ingreso del año 2013, en el primer periodo, al menos en once de ellas, un estudiante reprobó. Esto equivalió a un $68.8 \%$ de reprobación. Para el segundo y tercer periodo, así como en la calificación final de ciclo, dentro de todas las asignaturas se reportó al menos un estudiante reprobado.

Tabla 7. Porcentaje de aprobación y reprobación de Licenciatura en Ciencias de la Educación con Especialidad del Idioma Inglés

\begin{tabular}{|c|c|c|c|c|}
\hline \multirow{2}{*}{ Periodo } & \multicolumn{2}{|c|}{ Masculino } & \multicolumn{2}{c|}{ Femenino } \\
\cline { 2 - 5 } & Aprobados & Reprobados & Aprobados & Reprobados \\
\hline Uno & $42.3 \%$ & $57.7 \%$ & $63.4 \%$ & $36.6 \%$ \\
\hline Dos & $50.0 \%$ & $50.0 \%$ & $63.4 \%$ & $36.6 \%$ \\
\hline Tres & $65.4 \%$ & $34.6 \%$ & $82.9 \%$ & $17.1 \%$ \\
\hline Promedio Final & $65.4 \%$ & $34.6 \%$ & $75.6 \%$ & $24.4 \%$ \\
\hline
\end{tabular}

Nota: Se realizó una comparación entre las variables de periodo de estudio y sexo de los estudiantes, tomando información de los colectores de notas, UNICAES (2013).

Tabla 8. Porcentaje de aprobación y reprobación de Licenciatura en Enfermería

\begin{tabular}{|c|c|c|c|c|}
\hline \multirow{2}{*}{ Periodo } & \multicolumn{2}{|c|}{ Masculino } & \multicolumn{2}{c|}{ Femenino } \\
\cline { 2 - 5 } & Aprobados & Reprobados & Aprobados & Reprobados \\
\hline Uno & $100.0 \%$ & $0.0 \%$ & $90.6 \%$ & $9.4 \%$ \\
\hline Dos & $100.0 \%$ & $0.0 \%$ & $93.8 \%$ & $6.3 \%$ \\
\hline Tres & $33.3 \%$ & $66.7 \%$ & $81.3 \%$ & $18.8 \%$ \\
\hline Promedio Final & $100.0 \%$ & $0.0 \%$ & $93.8 \%$ & $6.3 \%$ \\
\hline
\end{tabular}

Nota: Se realizó una comparación entre las variables de periodo de estudio y sexo de los estudiantes, tomando información de los colectores de notas, UNICAES (2013). 
En detalle, los porcentajes de aprobación y reprobación, en cuanto a la calificación final de ciclo por asignatura, se muestran en la Tabla 9.

Tabla 9. Porcentajes de Aprobación y Reprobación por asignatura

\begin{tabular}{|c|c|c|}
\hline Asignatura & Aprobados & Reprobados \\
\hline Microbiología y Parasitología & $97.7 \%$ & $2.3 \%$ \\
\hline Bioquímica Humana & $97.1 \%$ & $2.9 \%$ \\
\hline Anatomía y Fisiología & $97.0 \%$ & $3.0 \%$ \\
\hline Epidemiologia & $96.4 \%$ & $3.6 \%$ \\
\hline Aprendizaje y Desarrollo Humano & $93.8 \%$ & $6.3 \%$ \\
\hline Fundamentos de Enfermería I & $93.8 \%$ & $6.3 \%$ \\
\hline Didáctica General I & $93.7 \%$ & $6.3 \%$ \\
\hline Teoría Gerencial II & $92.9 \%$ & $7.1 \%$ \\
\hline Informática & $88.2 \%$ & $11.8 \%$ \\
\hline Introducción al Marketing & $86.7 \%$ & $13.3 \%$ \\
\hline Ética Social & $85.8 \%$ & $14.2 \%$ \\
\hline Tecnología de la Información y Sociedad & $80.0 \%$ & $20.0 \%$ \\
\hline Teología II & $77.2 \%$ & $22.8 \%$ \\
\hline Intermediate English I & $77.1 \%$ & $22.9 \%$ \\
\hline Programación I & $62.5 \%$ & $37.5 \%$ \\
\hline Matemática II & $58.3 \%$ & $41.7 \%$ \\
\hline
\end{tabular}

Nota: Datos obtenidos de los colectores de notas y expresados en porcentajes, UNICAES (2013).

Por otra parte, se comparó la aprobación y reprobación de los estudiantes, tomando en cuenta la variable de las instituciones educativas de procedencia de los estudiantes (públicas y privadas):

\section{Alumnos aprobados y reprobados vs. variable institución de procedencia}

Figura 3. Gráfico con porcentajes de aprobación y reprobación de alumnos de acuerdo a la institución educativa donde cursaron bachillerato.

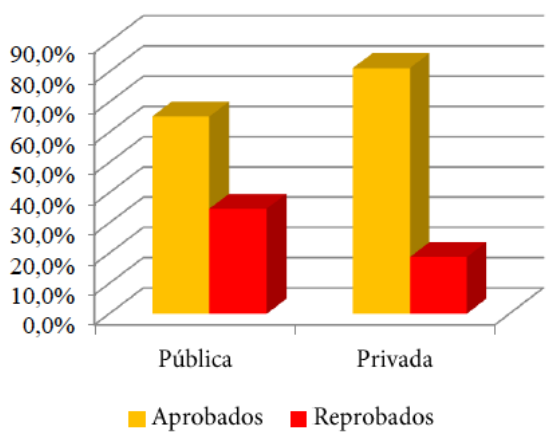


Al particularizar los resultados por departamento de residencia se obtuvo:

\section{Cantidad de alumnos aprobados y reprobados por departamento}

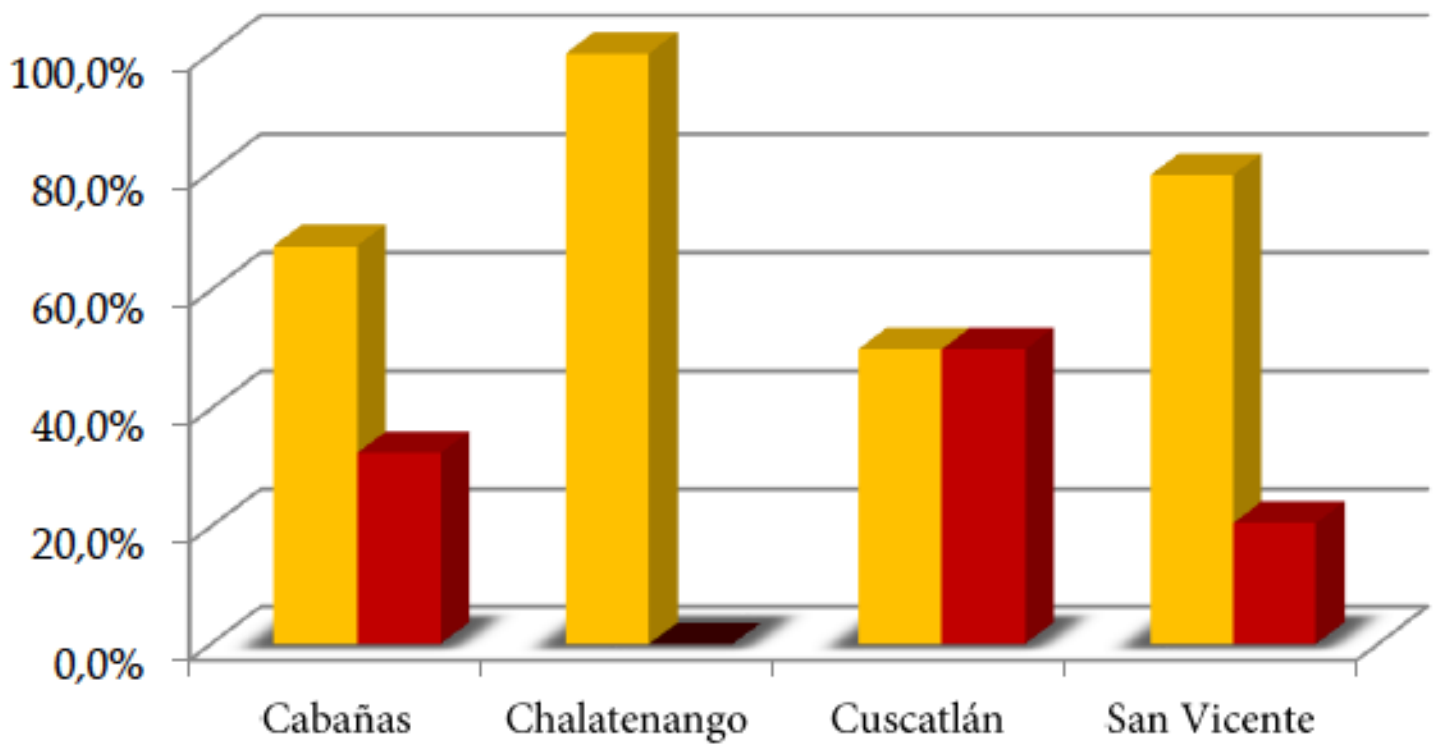

Aprobados $\square$ Reprobados

Figura 4. Gráfico con porcentajes de aprobación y reprobación de alumnos por Departamento de residencia.

Es necesario aclarar que, del departamento de Chalatenango, a la Universidad solo asiste un estudiante. Debido a esto, no existen suficientes datos para realizar alguna comparación.

Con la finalidad de detallar los resultados, se indagó sobre nombres de los institutos del cual provenían la mayoría de los estudiantes. A continuación se hace el desglose por departamento:

\begin{tabular}{|c|c|c|}
\hline Departamento & Cabañas & San Vicente \\
\hline \multirow{2}{*}{ Nombre de institutos $^{4}$} & Instituto Católico Karol & Instituto Nacional de \\
& Wojtyla (ICKW) & Apastepeque (INA) \\
& Instituto Nacional de & Instituto Nacional San José \\
& Ilobasco (INDI) & Verapaz (INSJV) \\
\hline
\end{tabular}

Figura 5. Institutos de procedencia de los alumnos de nuevo ingreso en el ciclo II-2013.

\section{Entiéndase, también, a través de sus diferentes siglas.}


En el caso de los institutos del departamento de Cuscatlán, al no existir una cantidad representativa de institutos específicos, no se tomaron en cuenta de manera individual.

Los porcentajes de aprobación y reprobación por instituto se muestran en la figura 5.

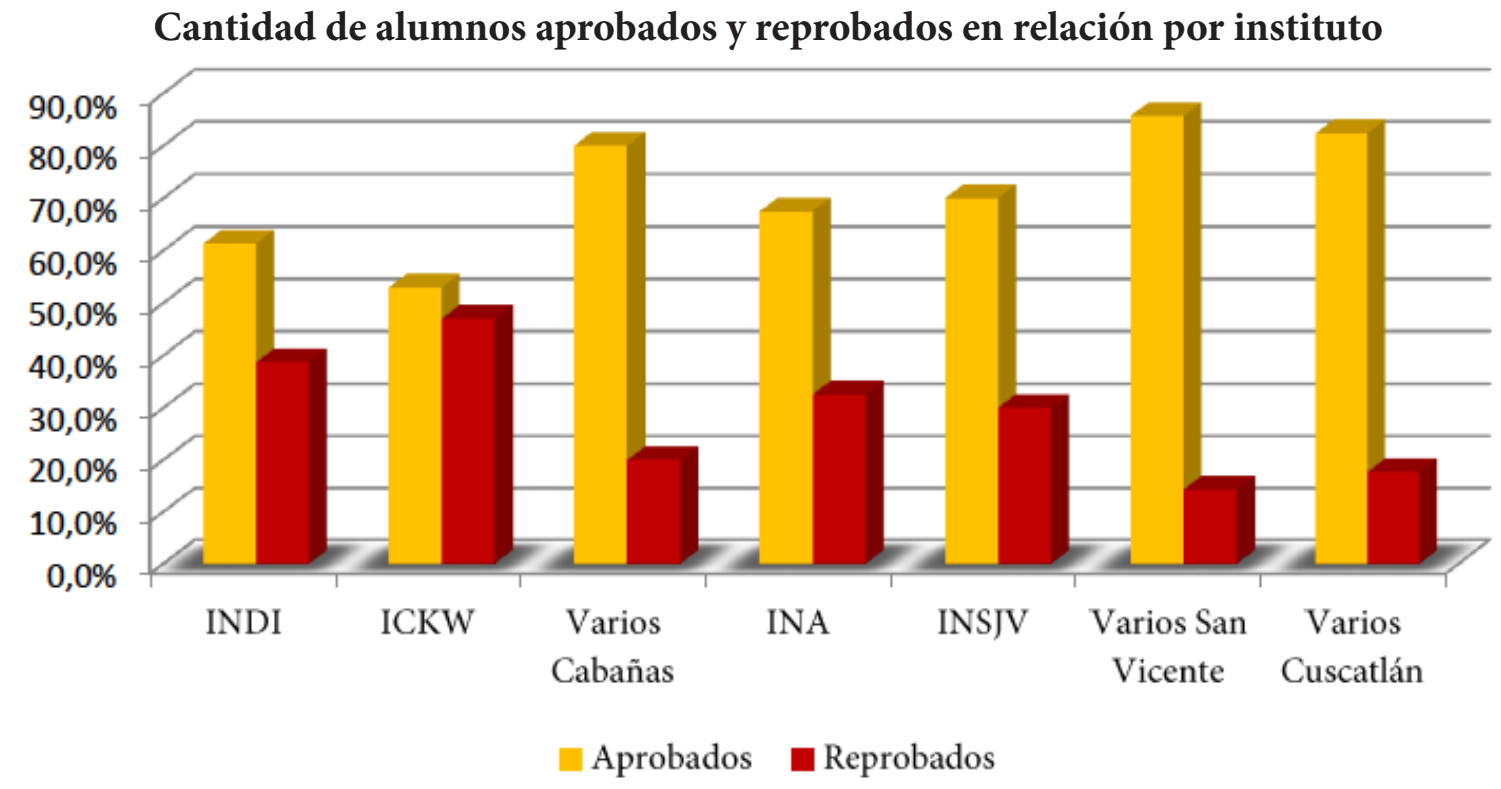

Figura 6. Gráfica de porcentaje de aprobación y reprobación de los alumnos por Instituto de procedencia.

\section{Discusión}

En términos generales, el $68.7 \%$ de los estudiantes aprobó todas sus asignaturas durante el ciclo II-2013.; dejando a un 31.3\% de alumnos reprobados con al menos una asignatura.

Existen diversas variables que afectan el rendimiento académico, por lo cual no se puede realizar un diagnóstico preciso sobre cuál o cuáles de ellas son las que más influyen en estos resultados. Sin embargo, una de las constantes estudiadas y que mostró un impacto significativo en el rendimiento académico fue el cambio de contexto o transición entre la Educación Media hacia la Educación Superior.
Ante este fenómeno, se indagó sobre los institutos de procedencia de los estudiantes y su ubicación geográfica, encontrándose que los alumnos provenientes de institutos del departamento de San Vicente presentan porcentajes de aprobación más altos. Estas cifras son seguidas por los alumnos provenientes de los departamentos de Cabañas y Cuscatlán. Un dato importante es que, la mayoría de la población estudiantil que se avoca a la Facultad Multidisciplinaria de Ilobasco pertenece al departamento de Cabañas. En cuanto a los institutos de los departamentos de San Vicente y Cabañas, Instituto Nacional San José Verapaz e Instituto Nacional de Ilobasco, respectivamente, ambos mostraron un alto porcentaje 
de estudiantes, que provenían de dichos centros educativos, y que aprobaron el ciclo.

Otro factor que afecta el rendimiento académico, y que fue señalado por los alumnos, fue el insuficiente tiempo que dedican al estudio, tomando en cuenta que no poseen conocimientos previos sobre las asignaturas que estudiarán en la Universidad. Algunas de las asignaturas en las que se detectaron porcentajes de reprobación mayores o similares fueron: Matemática II (41.7\%), Programación I (37.5\%), Intermediate English I (22.9\%), Teología II (22.8\%) y Tecnología de la Información y Sociedad (20\%). En el caso de Matemáticas, es notable relacionar este porcentaje con la nota promedio obtenida por los alumnos de los departamentos en estudio, dentro de la PAES (4.4). Esta realidad plantea un reto para las Instituciones de Educación Superior a nivel nacional, pero sobre todo regional.

No obstante, el rendimiento de los estudiantes mejoró en el transcurso del ciclo. Del total de la muestra, el $27.4 \%$ de los hombres mejoraron su rendimiento académico; dejando al sexo femenino con un 3.9\% de mejora. Esto dejó un alto porcentaje de aprobados (57.1\%) entre los hombres; $y$ por ende, un porcentaje bajo entre las mujeres (42.9\%) al final del ciclo.

Otro indicador que tiene relación con este aspecto es la relación docente-asignatura. Con cada docente y asignatura diferente, los estudiantes deben acoplarse al nivel de exigencia y la metodología que ello representa. Llama la atención que un $24 \%$ de los estudiantes entre el primer y segundo periodo afirmaron tener dificultadas para comprender al docente. Esto también estuvo aunado a la afinidad que los alumnos tenían hacia la carrera que cursaban. Algunos estudiantes manifestaron su deseo de cambiarse de carrera y/o perder interés en las asignaturas; a tal grado de retirarse definitivamente de la Universidad. No obstante, a nivel global, independiente de la carrera, el rendimiento académico de los estudiantes aumentó según avanzó el ciclo.

Entre otros factores, aunque en menor escala, se encontraron la falta de recursos económicos para continuar sus estudios y la inseguridad vivida en el trayecto de desplazamiento desde su hogar hacia la institución.

A manera de cierre, es importante que las variables identificadas que afectan el rendimiento académico sean atendidas y solventadas por las autoridades pertinentes, con la finalidad de potenciar un adecuado rendimiento académico entre los futuros estudiantes universitarios. 


\section{Referencias}

Artunduaga, M. M. (2008). Variables que influyen en el rendimiento academico en la universidad. Recuperado el 20 de septiembre de 2013, de Comision de Seguimiento, Orientación y Apollo para el Avance Académico de los Alumnos: http://www.ori.soa.efn.uncor. edu/?publicaciones=variables-que-influyen-en-el-rendimiento-academico-en-la-universidad

Daugherty, T. y Lane, E. (1999). A longitudinal study of academic and social predictors of college attrition. Social Behavior and Personality. Nueva Zelanda. 4 (27), 355-361.

Figueroa, C. (2004). Sistemas de Evaluación Académica. El Salvador: Editorial Universitaria.

Garvanzo V., G. M. (2007). Factores asociados al rendimiento académico en estudiantes universitarios, una reflexión desde la calidad de la educación superior pública. Revista Educación, 31(1), 43-63.

Girón, L. y González, D. (2005). Determinantes del rendimiento académico y la deserción estudiantil, en el programa de Economía de la Pontificia Universidad Javeriana de Cali. Economía, Gestión y Desarrollo. (3), 173-201.

Goméz, D., Oviedo, R., y Martinéz, E. (2011). Factores que influyen en el rendimiento académico del estudiante universitario. Recuperado el 16 de Octubre de 2013, de Tecnociencia Chihuahua: https://www.google.com.sv/search?q=\%22Factores+que+influyen+en+el+rendim iento+acad\%C3\%A9mico+del+estudiante+universitario\%22\&oq=\%22Factores+que+influyen + en+el+rendimiento+acad\%C3\%A9mico+del+estudiante+universitario\%22\&aqs=chrome..69 i57j0.2481j0j7\&sourceid

MINED. (1995). Ley de Educación Superior. San Salvador .

Ministerio de Educación de la Republica de El Salvador. (2013). Resultados PAES 2012. Recuperado el junio de 2013, de http://www.mined.gob.sv/jdownloads/Informes/resultados_ paes_2012_resumido.pdf

Navarro, R. E. (2003). El Rendimiento Académico: Concepto, Investigación y Desarrollo. Revista Electrónica Iberoamericana sobre Calidad, Eficacia y Cambio en Educación, 1(2).

Rodríguez, S., Fita, E., y Torrado, M. (2004). El rendimiento académico en la transición secundaria-universidad. Revista de Educación(334), 391-414. 
Salonava, M., Cifre, E., y Grau, R. (2005). Antecedentes de la autoeficacia en profesores y estudiantes universitarios: un modelo causal. Revista de Psicología del Trabajo y de las Organizaciones., 159-176.

Tejedor, F., y Ana, M. (2006). Causas del Bajo Rendimiento del Estudiante Universitario (En Opinión de los Profesores y Alumnos). Propuestas mejora en el marco del EEES. Recuperado el 27 de agosto de 2012, de Revista Educacion: http://www.revistaeducacion.mec.es/re342/ re342_21.pdf

Universidad Católica de El Salvador. (2013). Anuario académico. El Salvador. 